\title{
MODELO DE GESTIÓN EFICIENTE Y SOSTENIBLE DE LOS RECURSOS HÍDRICOS
}

\section{CHARACTERISTICS OF THE SYSTEMS TPM AND RCM IN THE MAINTENANCE ENGINEERING}

Carlos Tejedor Sánchez ${ }^{1}$

Francisco-Javier Cárcel-Carrasco ${ }^{2}$

1. Graduado en Arquitectura Técnica. Grupo Aguas de Valencia (España). E-mail: cartesan@arqt.upv.es

2. Doctor Ingeniero Industrial. ITM. Universitat Politècnica de València, Camino de Vera $S / N$, 46022, Valencia (España).E-mail: fracarc1@csa.upv.es

\section{Citación sugerida:}

Tejedor Sánchez, C. y Cárcel-Carrasco, F.J. (2017). Modelo de gestión eficiente y sostenible de los recursos hídricos. 3C Tecnología: glosas de innovación aplicadas a la pyme, 6(4), 13-23. DOI: $<$ http://dx.doi.org/10.17993/3ctecno.2017.v6n4e24.13-23/>. 


\section{RESUMEN}

El sector de distribución de agua potable a poblaciones está evolucionando mediante el uso de la innovación tecnológica. Las empresas gestoras de agua potable deben evolucionar los sistemas de gestión de sus redes. El desarrollo de herramientas de software, que se alimentan del Big Data proporcionado por la telelectura, está permitiendo alcanzar múltiples beneficios en materia medioambiental y de calidad del servicio. En este trabajo se muestra de una manera introductoria las características básicas y puntos de referencia para la realización de un modelo de gestión eficiente de los recursos hídricos, en base a la metrología de los contadores, los sistemas de telelectura, teniendo en cuenta el rendimiento hidraáulico y la verificación del parque de contadores. Todo ello se basa en las experiencias del grupo Aguas de Valencia en su experiencia e innovación en la gestión de recursos hídricos.

\section{ABSTRACT}

Distribution of drinking water to the population sector is evolving through the use of technological innovation. Managing drinking water utilities must evolve their network management systems. The development of software tools, which feed on Big Data provided by the remote reading, is allowing to achieve multiple environmental and service quality benefits. This work shows in an introductory manner the basic characteristics and benchmarks for the realization of a model of efficient management of water resources, on the basis of the Metrology of counters, remote reading systems, taking into account the performance hidraaulico and the verification of meters Park. All this is based on the experiences of the Aguas de Valencia group experience and innovation in the management of water resources.

\section{PALABRAS CLAVE}

Medición inteligente, Agua potable, Instalaciones de fontanería, Eficiencia hídrica.

\section{KEY WORDS}

Smart metering technologies, Potable water, Plumbing installations, Water efficiency. 


\section{INTRODUCCIÓN}

La Directiva Marco Europea del Agua (DMA) 2000/60/CE nace como respuesta a la necesidad de unificar las actuaciones en materia de gestión de agua en la Unión Europea. En línea de todo lo anteriormente expuesto, el incremento en la demanda y calidad del agua para todos los usos obliga a tomar las medidas necesarias que garanticen la sostenibilidad.

Tras un periodo de más de cinco años, entró en vigor en diciembre de 2000, participando un amplio grupo de actores del sector del agua en su redacción, sentando los principios fundamentales para una gestión eficiente y sostenible. En definitiva, esta Directiva plantea la modernización en la gestión de los recursos hídricos.

En líneas generales, los Estados miembros fomentan la participación activa de todas las partes interesadas por la aplicación de esta Directiva. Desde 2010, los Estados miembros deben garantizar que la política de tarificación incite a los consumidores a utilizar los recursos de forma eficaz y que los diferentes sectores económicos contribuyan a la recuperación de los costes de los servicios relacionados con el uso del agua, incluidos los costes medioambientales y de recursos. Estos objetivos se relacionan con este trabajo en el hecho de garantizar un uso sostenible del agua por parte de los particulares y las empresas (UNESCO, 2017).

Para afrontar estos importantes retos, es necesaria la modernización de los servicios de agua urbanos en el campo de la medición de consumos y recogida y gestión de la información (AENOR, 2014). Se ha reemplazado el concepto de lectura de contadores por el de gestión de datos o información, permitiendo alcanzar objetivos en el ahorro de agua, mejora del servicio o detección y corrección de fugas.

Es necesario que se busquen soluciones para integra los servicios de distribución pública de agua potabler y contribuir al avance de las Smart Cities o ciudades inteligentes.

En este artículo se muestra de una manera introductoria las características para la realización de un modelo de gestión eficiente de los recursos hídricos, en base a la metrología de los contadores, los sistemas de telelectura, teniendo en cuenta el rendimiento hidraáulico y la verificación del parque de contadores. Todo ello se basa en las experiencias del grupo Aguas de Valencia en su experiencia e innovación en la gestión de recursos hídricos (Aguas de Valencia, 2016; 2017).

\section{MODELO DE GESTIÓN}

El concepto de Smart City es muy extenso, engloba múltiples aspectos, pero está ineludiblemente ligado a la sostenibilidad en su máxima expresión. En las ciudades inteligentes, el objetivo es prestar el mejor servicio a cada ciudadano o usuario, buscando el máximo respeto por el entorno (Makropoulos, 2008). El desarrollo de esta idea inicial ha permitido que se subdivida en ramas especializadas que, integradas dentro del mismo ecosistema, dan forma a una ideología de desarrollo sostenible. 
La Asociación para la Innovación Europea sobre Ciudades y Comunidades Inteligentes (EIP-SCC) agrupa todos los actores para mejorar la vida urbana. Esto incluye el uso de las Tecnologías de la Información y la Comunicación (TIC).

Así mismo, AENOR ha dispuesto el Comité Técnico de Normalización sobre Ciudades Inteligentes (AEN/CTN 178), promovido por la Secretaría de Estado de Telecomunicaciones y para la Sociedad de la Información del Ministerio de Industria, Energía y Turismo (SETSI), para impulsar, racionalizar y optimizar la implantación en España de las ciudades inteligentes. La definición que propone dicho Comité es la siguiente:

"Ciudad Inteligente (Smart City) es la visión holística de una ciudad que aplica las TIC para la mejora de la calidad de vida y la accesibilidad de sus habitantes y asegura un desarrollo sostenible económico, social y ambiental en mejora permanente. Una ciudad inteligente permite a los ciudadanos interactuar con ella de forma multidisciplinar y se adapta en tiempo real a sus necesidades, de forma eficiente en calidad y costes, ofreciendo datos abiertos, soluciones y servicios orientados a los ciudadanos como personas, para resolver los efectos del crecimiento de las ciudades, en ámbitos públicos y privados, a través de la integración innovadora de infraestructuras con sistemas de gestión inteligente" (esmartcity, 2015).

Algunas de las ramas en las que se subdividen las Smart Cities son, por ejemplo: Smart Grids, o redes eléctricas eficientes; Smart Buildings, innovación en arquitectura y tecnología de la construcción; Smart Sensors, manteniendo toda la ciudad conectada; Smart Metering, y en concreto en materia de agua urbana, la gestión de la información proveniente de la telelectura. Es lo que actualmente se conoce como Internet de las Cosas (IoT).

En distintos congresos, el grupo Aguas de Valencia ha presentado este modelo de gestión como ideología para alcanzar la gestión eficiente y el desarrollo sostenible de los recursos hídricos. Los distintos eventos tienen en común esta filosofía, como el Congreso de Smart Cities de Vigo, las Jornadas Empresariales "Innovación, Cooperación e Internacionalización" de Castellón, Congreso Internacional Water Distribution System Analysis celebrado en Cartagena de Indias (Colombia), Foro del Agua de Los Cabos (México), el congreso "Leading Edge Conference on Water and Wastwater Technologies", organizado por la International Water Association (IWA) en Jerez de la Frontera, el Smart City Expo World Congress 2013 en Barcelona, o más recientemente la Jornada AEAS "Nuevos retos en la gestión de contadores en los abastecimientos" en el Oceanográfico de Valencia.

Recientemente, en noviembre de 2016, el Center For An Urban Future (CUF) y el Wagner Innovation Labs, de la Escuela Universitaria NYU Robert F. Wagner Graduate School of Public Service, de Nueva York, publicó un informe sobre 15 de las políticas urbanas más innovadoras lanzadas por ciudades de todo el mundo durante la última década, incluyendo el proyecto de Smart Metering de Aguas de Valencia. Destaca como "una ciudad importante está utilizando la tecnología de medición inteligente para reparar las fugas, detectar el fraude e influir en los residentes para reducir su consumo de agua", obteniendo "impresionantes resultados iniciales".

En resumen, mediante el uso de la innovación tecnológica, las empresas gestoras deben evolucionar los sistemas de gestión de agua urbanos. El desarrollo de varias herramientas de software, que se alimentan del Big Data proporcionado por la telelectura, está permitiendo a Aguas de Valencia alcanzar múltiples beneficios en materia medioambiental y de calidad del servicio. 


\section{METROLOGÍA DE LOS CONTADORES DE AGUA}

Según la Asociación Española de Fabricantes de Contadores de Agua (ANFAGUA), una parte importante de los contadores de uso doméstico en este país pueden estar midiendo, en mayor o menor medida, de forma errónea. Esta afirmación se basa fundamentalmente en la antigüedad de los parques de contadores, muy superior a la deseada. Es principalmente por este motivo por el que tratan de concienciar sobre la necesidad en la uniformidad de normativas sobre mantenimiento y renovación de los equipos de medida, estableciendo garantías metrológicas acordes a los objetivos marcados.

En la actualidad sólo está regulada la verificación primitiva, de obligado cumplimiento para la comercialización de los equipos. Serían aconsejables directrices normativas que regulasen la verificación periódica y después de una reparación, tal y como se hace en otros países (la exigencia mayor es en Alemania, cada 6 años). Este tipo de normas se aplican con mucho rigor en otros equipos de medida para suministros residenciales, como gas o electricidad, lo que todavía resulta más contradictorio.

La Directiva 2014/32/UE (Directiva sobre Instrumentos de Medida, MID) que deroga la antigua Directiva2004/22/CE, pese a que ésta es válida hasta el año 2016, define los requisitos y el carácter general que debe cumplir cualquier instrumento de medida y los requisitos específicos para cada tipo de instrumento. El RD 889/2006, por el que se regula el control metrológico del Estado sobre instrumentos de medida se ha visto derogado por el RD 244/2016 por el que se desarrolla la Ley 32/2014, de 22 de diciembre, de Metrología. Esta consiste en "el establecimiento y la aplicación del Sistema Legal de Unidades de Medida, así como la fijación de los principios y de las normas generales a las que debe ajustarse la organización y el régimen jurídico de la actividad metrológica en España".

La ley 32/2014, en su artículo 5 "Instrumentos o sistemas sometidos a control”, sí que hace hincapié en que "...los instrumentos, aparatos, medios y sistemas de medida que sirvan para pesar, medir o contar y que sean utilizados en aplicaciones de medida por razones de interés público, salud y seguridad pública, orden público, protección del medio ambiente, protección de los consumidores y usuarios, recaudación de impuestos y tasas, cálculo de aranceles, cánones, sanciones administrativas, realización de peritajes judiciales, establecimiento de las garantías básicas para un comercio leal y todas aquellas que puedan determinarse con carácter reglamentario, estarán sometidos al control metrológico del Estado..."(Centro Español de Metrología, 2016).

Entre algunas de las novedades se destaca:

- En la MID no se recogen disposiciones sobre la calibración de los equipos de medida, lo que supone que el período de calibración se seguirá regulando a nivel nacional.

- La clasificación de clases metrológicas se sustituye por la ratio Q3/Q1 (R)

- Se reorganizan las denominaciones métricas y caudales

- La MID define nuevos signos para los puntos de flujo:

1. Qmin se convierte en Q1 (caudal mínimo)

2. Qt se convierte en Q2 (caudal de transición)

3. Qn se convierte en Q3 (caudal nominal)

4. Qmax se convierte en Q4 (caudal máximo) 
Destaca entre las novedades el cambio en la denominación antes señalado, principalmente en lo que respecta a Qn y su "equivalente" Q3. La consecuencia directa sobre los millones de contadores con $\mathrm{Q}=1,5 \mathrm{~m}^{3} / \mathrm{h}$ de suministros residenciales es que se ha elevado a $\mathrm{Q} 3=2,5 \mathrm{~m}^{3} / \mathrm{h}$. Esto no supone que los contadores sean "más grandes" o que a partir de ahora estarían sobredimensionados para el mismo suministro, sino más bien que según la regulación de la MID estos contadores son capaces de registrar los caudales menores con mayor precisión. Por todo lo anteriormente comentado, en general la clasificación de los contadores según la MID obliga a someterse a unas exigencias más altas.

Desde ANFAGUA, haciendo mención al criterio de recuperación de costes de la DMA, inciden en que, si el contador registra con precisión el caudal utilizado por un usuario, éste abona el importe exacto determinado en la tarifa a la empresa gestora del abastecimiento, garantizando así la sostenibilidad del servicio.

Hay que apostar por la calidad metrológica, fijar unos estándares de precisión mínimos para los contadores de agua que garanticen una correcta medición de los consumos. Así, las ratios de los contadores empleados en sus abastecimientos se sitúan en valores mínimos de R160 con frecuencia, siendo el contador volumétrico principalmente el seleccionado.

\section{TELELECTURA}

Se define como telelectura a la lectura a distancia de los contadores. Se trata de añadir al contador tradicional un módulo de radio asociado que emite la señal de información, lo que permite disponer de 24 lecturas diarias y alarmas en el caso de haber algún tipo de fuga. Este tipo de sistema puede ser móvil o fijo.

En el sistema de comunicación en recorrido o Walk By (móvil), la lectura se realiza desde un vehículo o a pie, mediante el empleo de antenas, sin necesidad de acceder a los contadores. Para ello, se utiliza un terminal portátil con el software adecuado para la tecnología a leer. Este sistema es el más adecuado para contextos donde la dispersión entre los contadores es predominante. También se emplea este sistema para el mantenimiento de algunos de los equipos, así como en la transición entre la instalación de los mismos y la implantación de un sistema de lectura automático.

En el sistema de comunicación en red fija, no es necesaria la presencia de personal para realizar la lectura, que se lleva a cabo mediante una infraestructura de telecomunicaciones creada ad hoc para recolectar toda la información almacenada en los módulos de radiofrecuencia. Dicha infraestructura es la responsable de enviar toda esta información a los servidores centrales de Aguas de Valencia, donde se procesará toda esta información. Este sistema es el más recomendado para contextos donde la concentración de contadores predomina sobre los diseminados.

En función del tipo de tecnología empleada, la infraestructura se apoya en los diversos equipos de cada fabricante: gateways, repetidores, concentradores o cualquier otro equipo de similares características. Cada uno de los equipos de la red cumple una función específica para alcanzar el objetivo de recepción de lecturas en tiempo y forma deseados. En la figura 1, se representa el tratamiento que la información recibe desde el contador hasta los distintos servicios.

En las poblaciones con telelectura implantada, los concentradores reciben diariamente la información de los módulos, y la conservan hasta que posteriormente la envían al Gateway, equipo central de cada 
red que agrupa el total de la información y la envía a los servidores de Aguas de Valencia mediante tecnología 3G o GPRS.

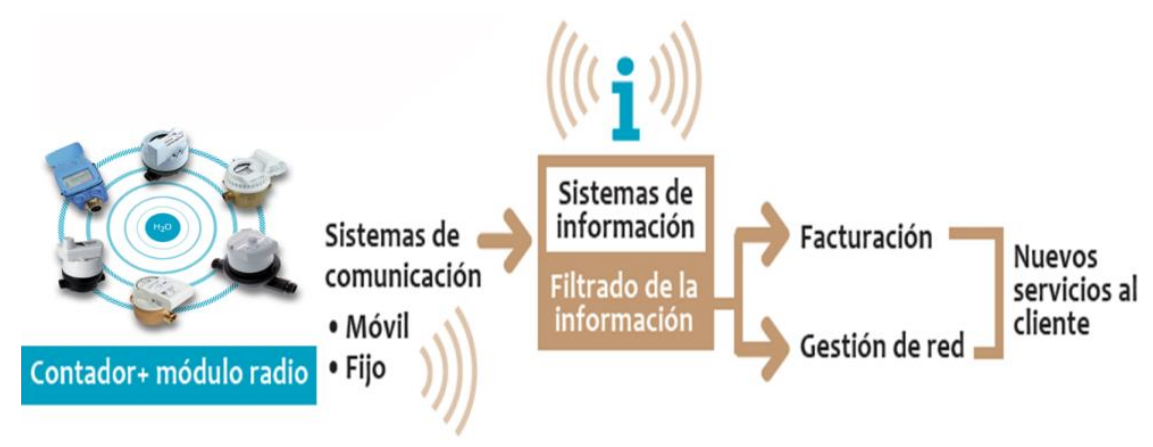

Figura 1. Esquema de tratamiento de la información. 2016. Aguas de Valencia.

La red fija es, por lo tanto, el objetivo final de la telelectura: la explotación total de la información para la mejora del servicio. La experiencia en este campo de Aguas de Valencia se extiende a más de 10 años, donde la trayectoria hacia esta solución ha sido firme. En la figura 2, se muestra la evolución de la telelectura por años, diferenciada por su método de lectura, así como el porcentaje de representación sobre el parque glogal.

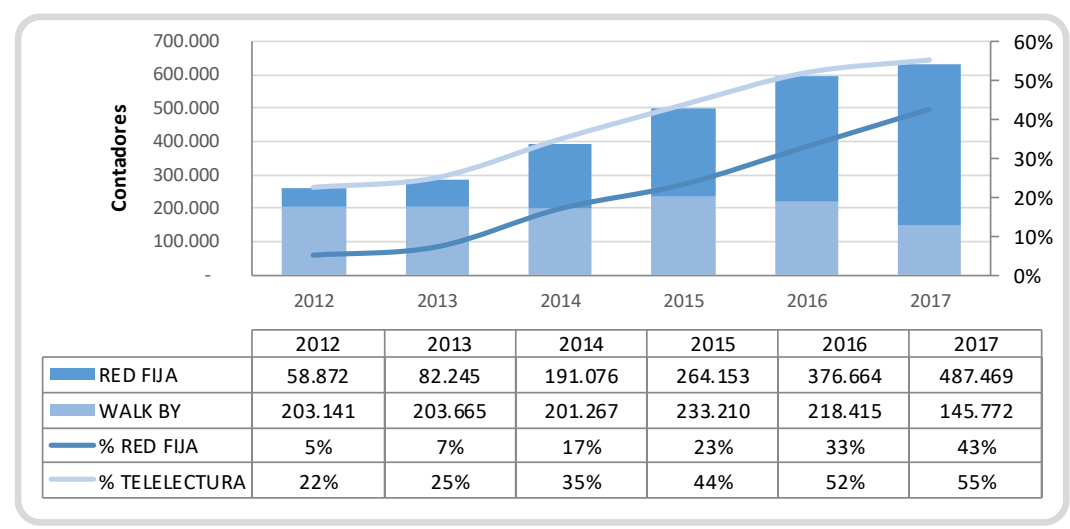

Figura 1. Evolución de la telelectura en Aguas de Valencia. 2017. Aguas de Valencia.

Actualmente, la cifra de contadores de Global Omnium con telelectura supera las 650.000 unidades, de las cuales más de 500.000 están integradas dentro de una infraestructura de red fija.

A lo largo de más de 10 años de experiencia, la apuesta por tecnologías multi-fabricante ha sido siempre un referente para Aguas de Valencia, ya que permite ofrecer a cada municipio una solución abierta y adaptable a sus necesidades concretas, eligiendo la más adecuada del mercado. Para alcanzar con éxito el desarrollo de esta idea, la empresa cuenta con un grupo especializado de ingenieros multidisciplinares integrando la Unidad de Telelectura. De este modo, mediante la especialización de un grupo de profesionales, es como se han podido obtener los resultados indicados.

Algunas de las ventajas que ofrece la telelectura son:

- Evita molestias a los clientes al no ser necesario acceder al interior de las viviendas

- No se realizan estimaciones de lecturas para facturar

- Proporciona a las industrias información detallada para la medición de huella hídrica

- Reducción de las reclamaciones de clientes hasta en un $60 \%$ desde su implantación

- Detección de consumos no autorizados 
- Establecimiento de patrones de consumo para la gestión de la demanda

- Proporciona la información detallada al cliente a través de la oficina virtual o mediante APP

- Detección de posibles fugas (700/mes)

- Aumento del rendimiento hídrico y eficiencia de la red mediante balances hídricos

- Adaptación de la producción a la demanda, ajustando la presión de la red y reduciendo fugas

- Beneficios medioambientales (reducción de 5,2 hm3/año y 1.400 TnCO2/año)

- Desarrollo de campañas de concienciación social sobre el consumo responsable

Algunas de las funciones más importantes que se realizan en la Unidad de Telelectura son:

- Presupuestos de instalaciones de redes fijas

- Selección de tecnología y diseño de instalaciones de redes fijas

- Monitorización básica de las redes fijas

- Monitorización avanzada de las redes fijas

- Mantenimiento de las infraestructuras de redes fijas

- Gestión incidencias de infraestructura de redes fijas con proveedores

Una vez definido el elevado volumen de datos que deben ser procesados, son necesarias multitud de herramientas integradas dentro de las Tecnologías de la Información y la Comunicación (TIC), para la gestión de datos, partes de trabajo, seguimiento de red, y relación con cllientes ( $\mathrm{W}$-mdM, W-mtM, Avalo, Merlin, Spot 3, Oficina virtual y APP móvil).

En conclusión, la telelectura es un elemento clave en la modernización de los sistemas de gestión de agua urbanos, conduciendo a una integración en las Smart Cities, y a un modelo de desarrollo sostenible y eficiente de los recursos hídricos. Como se ha citado anteriormente, lo que no se mide, no se puede mejorar (AWWA, 2003; 2017).

\section{RENDIMIENTO HIDRÁULICO}

Es necesario crear un departamento de Mejora del Rendimiento Hidráulico enmarcado en la filosofía de gestión eficiente y sostenible, con el claro objetivo de reducir las pérdidas de agua en las redes de distribución (pérdidas reales), ahorrando no sólo agua, sino también energía. La mejora de la eficiencia hidráulica, se refleja en varios indicadores, siendo el balance hídrico el de mayor interés en este estudio.

Todas estas fugas de agua en las redes de distribución se pueden dar en canalizaciones, pero también en uniones, válvulas, acometidas, bocas de riego y de incendios, o cualquier otro elemento de la infraestructura. Es importante destacar que el $70 \%$ de las pérdidas reales no se manifiesta en la superficie. Como ejemplo, indicar que una perforación en una tubería de apenas $1 \mathrm{~cm}^{2}$, con una presión cercana a $35 \mathrm{mca}$, pierde alrededor de $4 \mathrm{~m} 3 / \mathrm{h}$. Este valor equivale, en el caso de no localizarse y repararse, a la pérdida de $35.000 \mathrm{~m}^{3}$ anuales, equivalente a una población de 550 habitantes. En costes energéticos, $14.000 \mathrm{~kW}, 9.000 \mathrm{~kg} \mathrm{CO} 2$.

Se debe intensificar los esfuerzos técnicos y económicos mediante la activación de este grupo de Mejora del Rendimiento Hidráulico. Las actuaciones se deben cimentar sobre 4 pilares, siguiendo las recomendaciones del IWA: 
- Gestión de la presión: mediante la instalación de válvulas reguladoras. Existe una relación directa, como se ha planteado en el ejemplo anterior, entre la presión y el caudal fugado.

- Control del caudal mínimo nocturno: instalando registradores de caudal y presión. Es muy importante definir con precisión este parámetro, ya que es un claro indicador de fugas. El objetivo es controlar la gestión de la demanda.

- Mejora de las infraestructuras: la renovación de las canalizaciones y otros elementos de la red, así como las infraestructuras de telelectura propiamente dichas, complementan los esfuerzos destinados a la eficiencia de la red.

- Búsqueda de fugas: a través de un equipo de trabajo muy especializado, que utiliza las tecnologías más modernas disponibles en el mercado para la localización de fugas, ya sean medios acústicos (prelocalizadores, correladores, geófonos o hidrófonos) o de otro tipo (termografía, cámaras de inspección).

Para poder cumplir con estos exigentes objetivos, la red se desglosa en varios componentes, sectores, registradores, contadores y caudalímetros, válvulas reguladoras de presión, canalizaciones y acometidas. Se registran los caudales inyectados en la red y los consumidos y se realizan actuaciones diarias sobre las instalaciones.

La gestión de toda esta información, en el caso del grupo Aguas de Valencia, se lleva a cabo con el software de desarrollo propio denominado Merlín, que importa la información de registradores y estaciones remotas, así como la telelectura por red fija, para todos los cálculos diarios que realiza.

En resumen, se debe trabaja activamente en la gestión de las pérdidas de agua en las redes de abastecimiento hidráulico, como una de las principales vías para alcanzar la eficiencia en la gestión.

\section{VERIFICACIÓN DEL PARQUE DE CONTADORES}

Dada la importante cantidad de información que se recibe con la implantación de redes fijas de telelectura, es necesario estructurar el tratamiento de la misma para que los resultados sean eficaces y útiles.

Como ejemplo del incremento en la recepción de información, en la etapa previa a la telelectura en una pequeña población, se recibían alrededor de 1.500 .000 lecturas anuales. Esta cifra ha crecido exponencialmente hasta superar las 1.600.000.000 lecturas en un año. A esta cantidad hay que sumar la información procedente de las alarmas que cada tecnología es capaz de procesar. Por citar algunas de ellas, se reciben diariamente alarmas sobre posibles fugas, detección de caudales inversos, manipulación no autorizada del contador de agua, posible fraude magnético, etc.

Ante el progresivo aumento de la información a procesar y ante la el hecho de que se reducen las visitas sistemáticas a las instalaciones, surge la necesidad de crear una Unidad específica responsable de las tareas derivadas del tratamiento de esta información. Así, la Unidad de Verificación del Parque de Contadores debe realizar las siguientes funciones:

- Detección de fraudes a partir de las alarmas que generan los módulos de radio en contadores

- Detección de fraudes a partir de la verificación sistemática en campo de suministros candidatos como son los casos de bajas por falta de pago o muestreos en el resto de casos 
- Detección y verificación en campo de fraudes a partir del análisis de la información de las lecturas (principalmente en red fija) mediante algoritmos.

- Detección y verificación en campo de situaciones anómalas obtenidas a partir del análisis de la información de las lecturas (principalmente en red fija) mediante algoritmos

- Verificación de consumos nulos en contadores con telelectura para determinar si el contador está parado o no habitan en la vivienda o no hay actividad en la industria.

- Verificación de consumos en suministros en baja en los que no se ha retirado el contador

- Seguimiento del cumplimiento y ejecución de las órdenes de trabajo críticas

En consecuencia, es la unidad de verificación del parque de contadores la que tiene la responsabilidad de materializar en resultados todos los avisos obtenidos del tratamiento de la información de la red fija. A medida que ha ido aumentado la experiencia dentro de Aguas de Valencia, los algoritmos empleados para el análisis de la información se han ido optimizando, permitiendo en la actualidad alcanzar una elevada tasa de éxito, y en consecuencia un aprovechamiento cada vez mayor de las infraestructuras implantadas (ANFAGUA, 2017).

\section{CONCLUSIONES}

Mediante el uso de la innovación tecnológica, las empresas gestoras de agua potable en poblaciones deben evolucionar los sistemas de gestión de agua urbanos. El desarrollo de varias herramientas de software, que se alimentan del Big Data proporcionado por la telelectura, está permitiendo alcanzar múltiples beneficios en materia medioambiental y de calidad del servicio.

Actualmente sólo está regulada la verificación primitiva de contadores de agua, de obligado cumplimiento para la comercialización de los equipos. Serían aconsejables directrices normativas que regulasen la verificación periódica y después de una reparación, tal y como se hace en otros países (la exigencia mayor es en Alemania, cada 6 años). Este tipo de normas se aplican con mucho rigor en otros equipos de medida para suministros residenciales, como gas o electricidad, lo que todavía resulta más contradictorio.

La telelectura es un elemento clave en la modernización de los sistemas de gestión de agua urbanos, conduciendo a una integración en las Smart Cities, y a un modelo de desarrollo sostenible y eficiente de los recursos hídricos. Hay que actuar ante la filosofía de que lo que no se mide, no se puede mejorar.

Los departamentos de mejora del rendimiento hidráulico deben basarse en la filosofía de gestión eficiente y sostenible, con el claro objetivo de reducir las pérdidas de agua en las redes de distribución (pérdidas reales), ahorrando no sólo agua, sino también energía. La mejora de la eficiencia hidráulica, se refleja en varios indicadores, siendo el balance hídrico el de mayor interés.

Debe existir una unidad de verificación del parque de contadores con la responsabilidad de materializar en resultados todos los avisos obtenidos del tratamiento de la información de la red fija. 


\section{REFERENCIAS BIBLIOGRÁFICAS}

AENOR (2014): El papel de las normas en las ciudades inteligentes - Informes de Normalización. Madrid: Comité AEN/CTN 178.

Aguas de Valencia se sitúa a la cabeza de la tecnología de telelectura de contadores. (2016). economía3. Disponible en: http://www.economia3.com/2016/06/20/77027-aguas-de-valenciase-situa-a-la-cabeza-de-la-tecnologia-de-telelectura-de-contadores/

Aguas de Valencia, pionera en el uso del «big data». (8 de 1 de 2017). levante-emv. Disponible en: http://www.levante-emv.com/suscriptor/mercantil-valenciano/2017/01/08/aguas-valenciapionera-big-data/1512541.html

ANFAGUA. (2017). Recomendaciones generales de instalación de los contadores de agua. Disponible en: http://anfagua.org/informacion-tecnica/requisitos-y-recomendaciones/condiciones-deinstalacion

AWWA, \& IWA. (2003). Applying Worldwide Best Management Practices in Water Loss Control. Disponible en: https://www.awwa.org

AWWA (american water works association). 2017. Disponible en: https://www.awwa.org/

Esmartcity. (2015). La telelectura, base del abastecimiento inteligente de agua de una Smart City. Disponible en: https://www.esmartcity.es/comunicaciones/telelectura-base-abastecimientointeligente-agua-smart-city

Makropoulos, C.K., Nastis, K., Liu, S., Mittas, K., Butler, D. 2008. Decision support system for sustainable option selection in integrated urban water management. Environmental Modelling \& Software, 23(12), 1448-1460. doi:10.1016/j.envsoft.2008.04.010.

UNESCO. (2017). Programa Mundial de Evaluación de los Recursos Hídricos (WWAP). Disponible en: http://www.unesco.org/new/es/natural-sciences/environment/water/wwap/facts-andfigures/all-facts-wwdr3/fact1-demographics-consumption/ 